\title{
AN UNUSUAL PRESENTATION OF GASTRIC DIVERTICULUM: A RARE FORM OF DIVERTICULUM OF THE GASTROINTESTINAL TRACT
}

\section{Gabriela Jungblut Schuh', Sandra Jungblut Schuh², Ivan Morzoletto Pedrollo ${ }^{3}$, Antônio Carlos Maciel $^{2}$}

\section{INTRODUCTION}

Clin Biomed Res. 2020;40(1):58-60

1 Faculdade de Medicina, Universidade Federal do Rio Grande do Sul (UFRGS). Porto Alegre, RS, Brasil.

2 Departamento de Radiologia e Diagnóstico de Imagem, Hospital Santa Casa de Misericórdia de Porto Alegre. Porto Alegre, RS, Brasil.

3 Departamento de Radiologia e Diagnóstico de Imagem, Hospital Moinhos de Vento. Porto Alegre, RS, Brasil.

Corresponding author: Gabriela Jungblut Schuh gschuh@hcpa.edu.br

Faculdade de Medicina, Universidade Federal do Rio Grande do Sul (UFRGS)

Rua Ramiro Barcelos, 2400. 90035-002, Porto Alegre, RS, Brasil.
We report two cases of gastric diverticulum (GD) documented by upper gastrointestinal contrast radiographic studies and computed tomography (CT). The first case is a 51-year-old woman with a typical presentation of GD occurring on the posterior wall of the fundus (Figure 1). The second case is a 72-year-old woman with sliding diaphragmatic hernia. In this patient, the gastric fundus, which also has a diverticulum, is herniated and located inside the thorax (Figure 2 and Figure 3). Both patients discovered the presence of $\mathrm{GD}$ incidentally during diagnostic testing for other reasons.

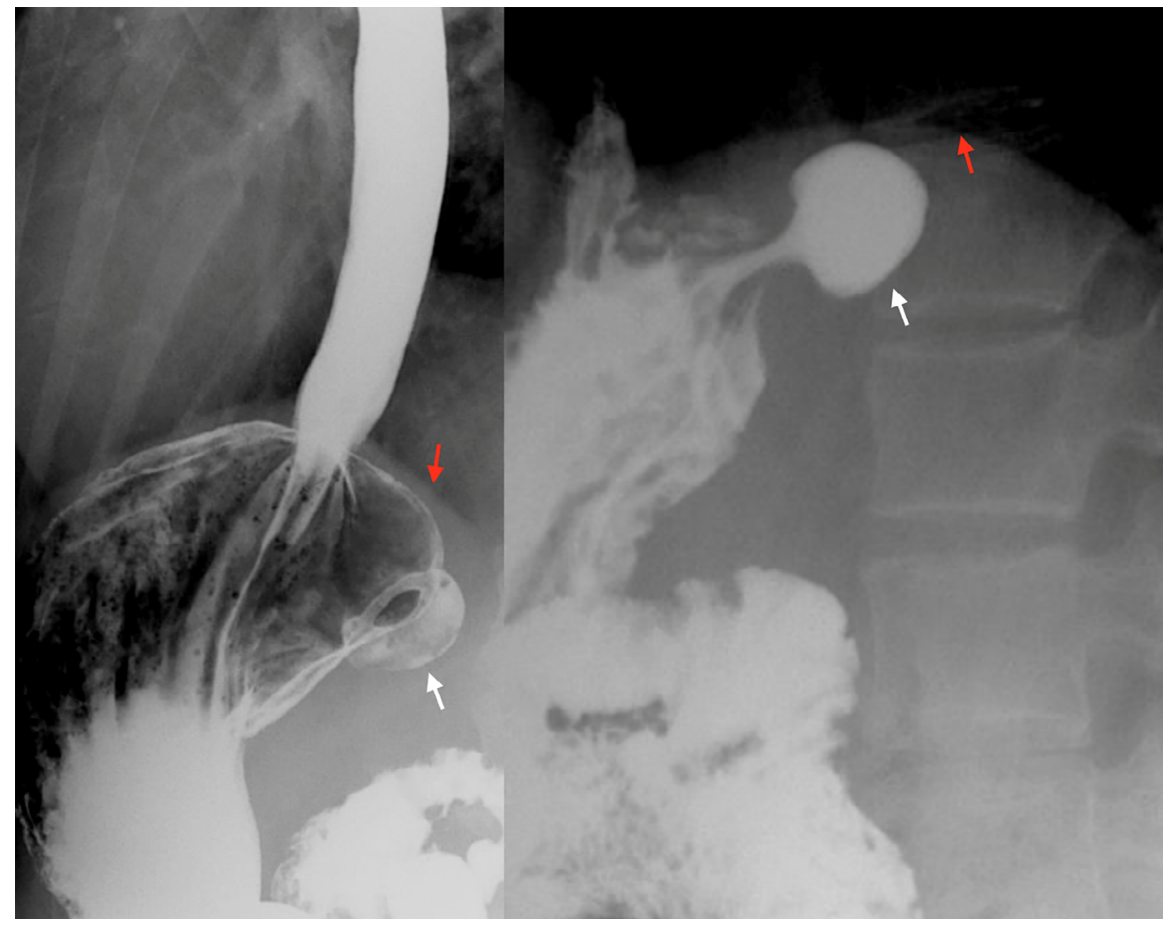

Figure 1: Contrast radiograph of the esophagus, stomach and duodenum showing a gastric diverticulum (white arrows) on the posterior wall of the fundus, the most common location. Red arrows: diaphragm. 


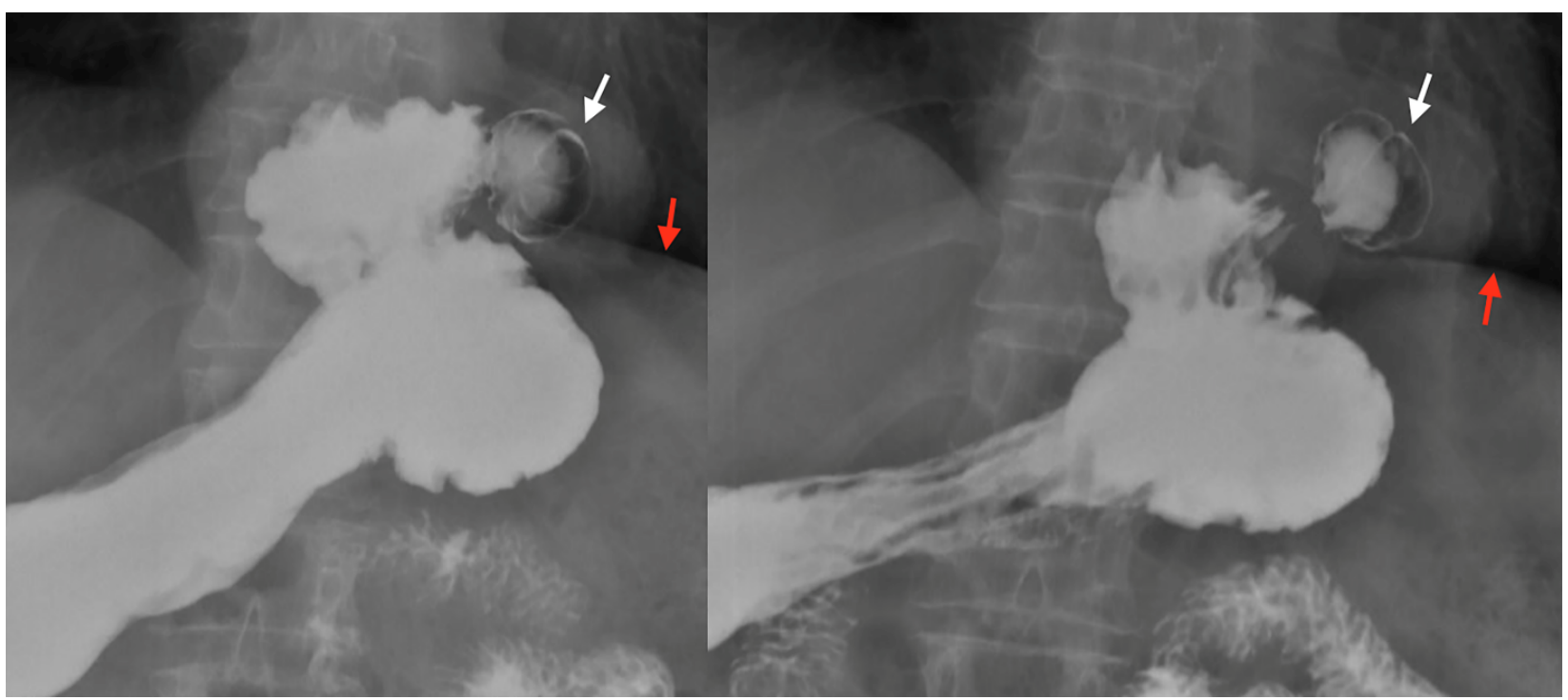

Figure 2: Contrast radiograph of the esophagus, stomach and duodenum showing a large hiatus hernia. The gastric fundus, which has a diverticulum (white arrows), is herniated and located inside the thorax. Red arrows: diaphragm.

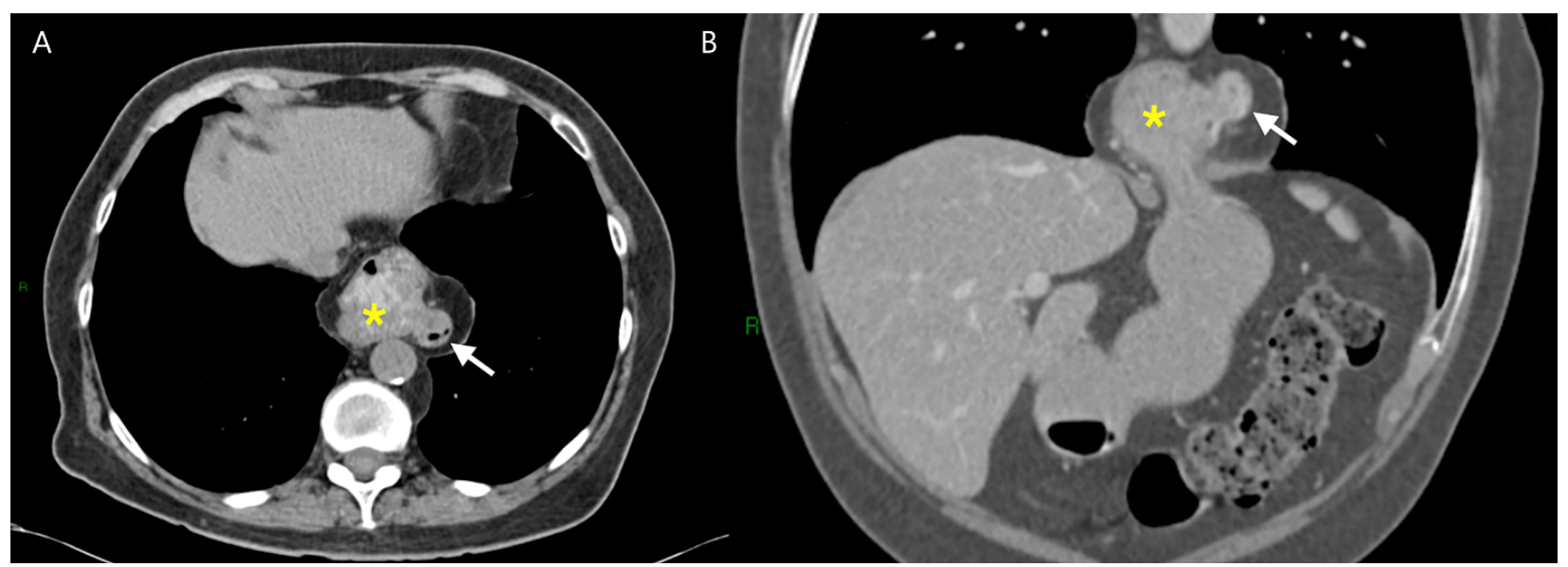

Figure 3: A: CT scan with axial; B: and coronal reconstruction; C: images showing a hiatus hernia (asterisks) and a gastric diverticulum (white arrow).

\section{DISCUSSION}

A diverticulum can develop throughout the gastrointestinal tract and takes the form a pouch structure projecting outward from the canal that may contain one or more of the gastrointestinal tract layers ${ }^{1}$. The purpose of this report is to discuss diverticular formation in the stomach, which is rare $^{2}$ and the least common type of gastrointestinal diverticula ${ }^{3}$. Detection rates vary with the study method, ranging from $0.02 \%$ in autopsy studies to $0.04 \%$ in upper gastrointestinal contrast radiographic studies and $0.01-0.11 \%$ in upper gastrointestinal endoscopies ${ }^{3}$. Gastric diverticulum (GD) typically presents between the fifth and seventh decades of life, with equal distribution among men and women ${ }^{3,4}$. However, GD can occur at any age, with cases reported in newborns ${ }^{4}$.
In $75 \%$ of cases, GD occurs on the posterior wall of the fundus ${ }^{2}$. Fundal diverticula often arise near the esophagogastric junction ${ }^{5}$. They are usually solitary and have a small orifice and short neck, although they may reach a considerable size ${ }^{2}$, ranging from $2 \mathrm{~cm}$ to $10 \mathrm{~cm}^{5}$. A fundal diverticulum can be easily identified by its unique appearance, pathognomonic signs, and constant location ${ }^{5}$. The second most common location is the prepyloric area, with about $15 \%$ of occurrences along the greater curvature. Usually small and solitary, these diverticula may not be apparent on the serosal surface at operation and have been termed partial or intramural diverticula ${ }^{2}$.

GD can be classified into two types: congenital or true diverticula, which contain all layers of the gastric wall; and acquired or false diverticula, which can be further classified as either traction or pulsion 
diverticula and are composed of mucosa and submucosa. While pulsion diverticula are usually caused by increased intraluminal pressure (e.g., chronic cough), traction diverticula are derived from adjacent inflammatory processes or perigastric adhesions of coexisting lesions (e.g., peptic ulcer disease, pancreatitis, Roux-en-Y gastric bypass surgery $)^{3}$. The most common type of GD, fundal diverticulum, has been traditionally considered to represent congenital diverticula ${ }^{5}$.

\section{Conflicts of Interests}

The authors declare no conflicts of interest.

\section{REFERENCES}

1. Sinclair A. Diverticular disease of the gastrointestinal tract. Prim Care. 2017;44(4):643-54. doi: 10.1016/j. pop.2017.07.007

2. Putman CE, Ravin CE. Textbook of diagnostic imaging. 2nd ed. Philadelphia: W. B. Saunders; 1994.
3. Shah J, Patel K, Sunkara T, Papafragkakis C, Shahidullah A. Gastric diverticulum: a comprehensive review. Inflamm Intest Dis. 2019;3(4):161-6. doi: $10.1159 / 000495463$
Fundal diverticula are usually asymptomatic ${ }^{2}$ and discovered incidentally during routine diagnostic testing. However, they can also present with varying abdominal symptoms, including dyspepsia, vomiting and abdominal pain. Occasionally, complications such as ulceration, perforation, hemorrhage and malignancy may develop. These complications may be life-threatening and are indications for surgical management. There is no specific treatment for an asymptomatic diverticulum ${ }^{3,4}$.
4. Hajini FF, Husain M, Bhat A, Bukhari SI Gastric diverticulum a rare endoscopic finding. BMJ Case Rep. $2014 \mathrm{Apr}$ 1;2014. doi: 10.1136/bcr-2013-202887
5. Margulis AR, Burhenne HJ, editors. Practical alimentary tract radiology. St. Louis: Mosby Year Book; 1993.

Received: Jan 23, 2020 Accepted: Mar 29, 2020 